\title{
Ways to improve physico-mechanical properties of polymer composites on the basis of secondary polypropylene and natural extenders
}

\author{
A. R. Sadritdinov, R. Y. Lazdin, E. M. Zakharova, A. S. Shurshina, V.P. Zakharov, E. I. Kulish ${ }^{\dagger}$ \\ †onlyalena@mail.ru
}

Bashkir State University, 32 Zaki Validi str., Ufa, 450076, Russia

\begin{abstract}
Development of polymer composite materials is one of the most promising directions of the modern polymer materials science. Compatibility of components, for example, a polymer and an extender can cause problems in the development of such systems. To provide connectivity of components, the composite structure is extended with coupling agents. The effect of compatibilizers on the rheological and physico-mechanical behavior of a polymer compound based on the secondary polymeric raw materials extended with vegetable components is studied in this paper. It is shown that extending the composition with compatibilizers affects the melt flow index (MFI) values - the more compatibilizer there is in the composition, the higher the MFI value is, and therefore, the better its workability. At the same time, it should be noted that in case of using Kompplen EN13F1F3HH and San UMB UGOF1 as compatibilizers the regularities of the MFI change are different - the increase of MFI values is observed only when the content of the compatibilizer in the composition amounts to $1 \% \mathrm{wt}$. As evidenced by rheological data, adding even a small amount of a compatibilizer results in less pronounced resilient properties of the composition containing the modifier, which reduces the possibility of manufacturing defects. Extending a composition with modifiers also affects its physico-mechanical properties. The effect of San UMB UGOF1 and Kompplen EN13F1F3HM modifiers is similar, while the one of Kompplen MPP5X differs. The lowest values of the elastic modulus are registered when using San UMB UGOF1 modifier. At the same time, the increase in the extender content in the composition reduces the elastic modulus, which has a low value even without that. Thus, as part of the study, the principal possibility to regulate the rheological and physicomechanical behavior of polymer composites not only by extending them with compatibilizers acting as a surface active substance but also by regulating the production conditions is shown.
\end{abstract}

Keywords: secondary polymers, natural extender, coupling agents.

\section{Introduction}

Nowadays, the development of polymer composite materials is one of the most promising directions of the modern polymer materials science [1-3]. Of special interest are the so called biodegradable and biodecomposed plastics and composite materials that after using degrade into components safe for the environment [4-10]. The properties of such materials can be varied widely depending on the polymeric matrix used, the type of the extender, its dispersity and concentration. It is necessary to be aware of the regularities of the extenders effect on the properties of polymers in order to produce materials with tailor-made properties by extending them with disperse particles. Although the fact that the polymer extended with disperse particles is a twophase system consisting of a polymer disperse medium and particles of the hard disperse phase often with no affinity between them makes it challenging.

If a polymer and an extender are incompatible, the work of adhesion between the polymer and disperse particles is quite low. In this case the material obtained has reduced mechanical properties. With the increase in the work of adhesion the distribution of the load applied to the material is more or less uniform without a significant stress concentration on the polymer-extender boundary. It is possible to obtain composites with relatively high mechanical properties at high strength of the polymer-extender bonding.

Unfortunately, the molecules of the most widely used polymers of the polyolefin group have poor compatibility with particles of vegetable extenders. The polymers are hydrophobic and non-polar, and such extenders as groundwood are, on the contrary, hydrophylic and polar. For this reason, the result of a standard extending of the polyolefin matrix with such extenders is not a composite but a physical mixture of the extender and the polymer with poor mechanical strength characteristics and low environmental resistance. To provide adhesion of the components of the composite, it is extended with special additives - coupling agents - immediately in the process of production [11-14]. The substances exhibit surface-active properties and are diphilic, which contributes to their compatibility with a lot of polymers and intensifies interfacial interaction and, consequently, affects the physicomechanical properties of the composite.

The objective of the present work is to study the effect of extenders on the rheological and physico-mechanical properties of a polymer compound based on the secondary polymeric raw materials extended with vegetable components. 


\section{Experiment}

A sample of secondary polypropylene (SPP) with properties corresponding to $\mathrm{FF} / 3350$ virgin polypropylene (LLC "ZPI Alternativa” (Russia, Republic of Bashkortostan, Oktyabrskiy city) was used in the experiment. Rice hulls (RH) and groundwood (GW) were used as extenders for polymer compounds. Before mixing the extender was dried in a heat chamber at $100^{\circ} \mathrm{C}$ for 5 hours. Kompplen EN13F1F3HH, Kompplen MPP5X and San UMB UGOF1 (LLC "KomPlast", Protvino) were used as compatibilizers. Kompplen EN13F1F3HH is a rubber-like ethylene with maleic anhydride and methacrylate graft (0.9-1.5\%), San UMB UGOF1 is a maleic anhydride grafted ethylene (0.9\%), Kompplen MPP5X is ethylene methylacrylate with peroxy groups.

The composite materials were obtained using «PlastographEC» (Brabender, Germany) for 15 minutes at the load $200 \mathrm{~N}$, temperature $180^{\circ} \mathrm{C}$ and screw speed 30 revolutions per minute. The pressing was done using automatic hydraulic press «AutoMH-NE» (Carver, USA) at varied temperature, applied force and cooled/uncooled plates. The following pressing modes were tested:

mode $1-$ force $7000 \mathrm{kgf}$, plates temperature $180^{\circ} \mathrm{C}$, uncooled;

mode $2-$ force $7000 \mathrm{kgf}$, plates temperature $180^{\circ} \mathrm{C}$ cooled;

mode 3 - force $7000 \mathrm{kgf}$, plates temperature $210^{\circ} \mathrm{C}$, uncooled;

mode $4-$ force $7000 \mathrm{kgf}$, plates temperature $210^{\circ} \mathrm{C}$, cooled;

mode 5 - force $15000 \mathrm{kgf}$, plates temperature $180^{\circ} \mathrm{C}$, uncooled;

mode 6 - force $15000 \mathrm{kgf}$, plates temperature $180^{\circ} \mathrm{C}$, cooled;

mode 7 - force $15000 \mathrm{kgf}$, plates temperature $210^{\circ} \mathrm{C}$,

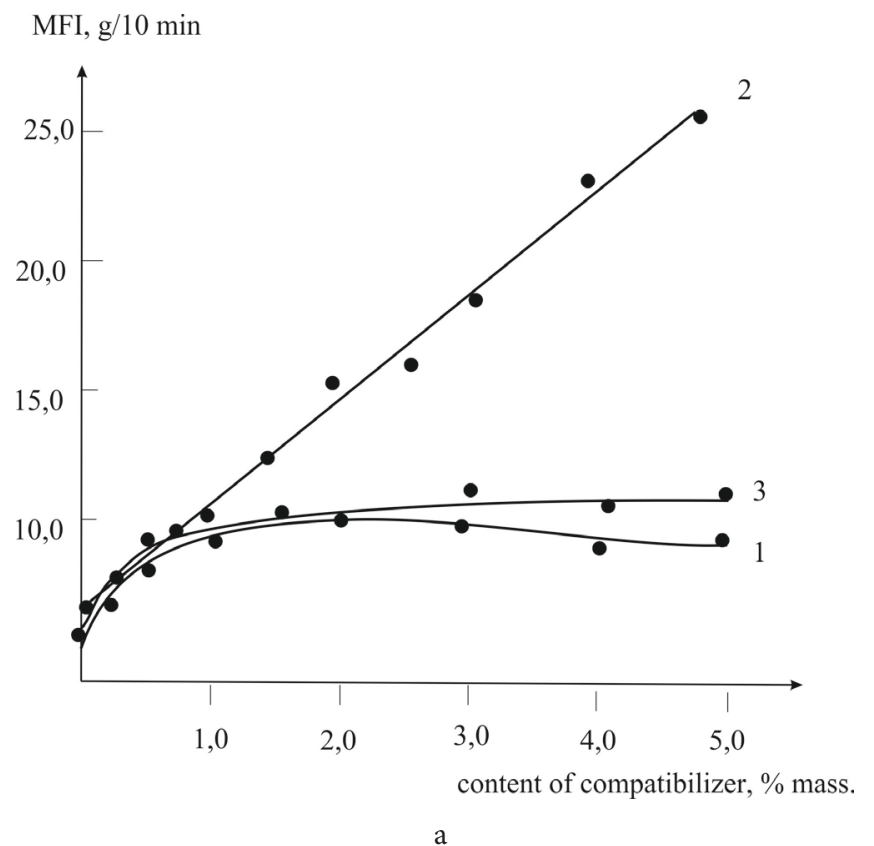

uncooled;

mode 8 - force $15000 \mathrm{kgf}$, plates temperature $210^{\circ} \mathrm{C}$, cooled.

The physico-mechanical properties of the polymer composites were determined according to the Russian standard GOST 11262-80 using breaking machine «ShimadzuAGS-X» (Shimadzu, Japan). The melt flow index (MFI) was determined for the $2.16 \mathrm{~kg}$ weight at $190^{\circ} \mathrm{C}$ using a melt flow index meter. Rheological measurements were done in the oscillation mode within the oscillation frequency range from 0.01 to $100 \mathrm{~Hz}$ at $220^{\circ} \mathrm{C}$ using Haake MarsIII modular dynamic rheometer.

\section{Results and discussion}

As evidenced by the analysis of the plastograms, the maximum torque increases sharply and amounts to $M_{\max }$ value at the initial point of time, which shows the energy consumption during the components mixing process, then the value reaches equilibrium. It should also be noted that the values of the maximum torque were the largest in the region of low concentrations of the compatibilizer with $0.5-1 \%$ wt. of the SPP content. The increased interaction between the components of the composite appears to occur at low compatibilizer content.

Extending the composition with the compatibilizer has an effect on MFI values as well. As is seen in Fig. 1, the more compatibilizer there is in the composition, the higher the MFI value is and, therefore, the lower are the composition viscosity and better its workability. However, when using Kompplen EN13F1F3HH and San UMB UGOF1 as compatibilizers the increase of the compatibilizer content in the system by more than $1 \%$ wt. is practically not followed by changes in MFI values.

Since the evaluation of thermoplasts by MFI value is key to the technological process recommended for processing

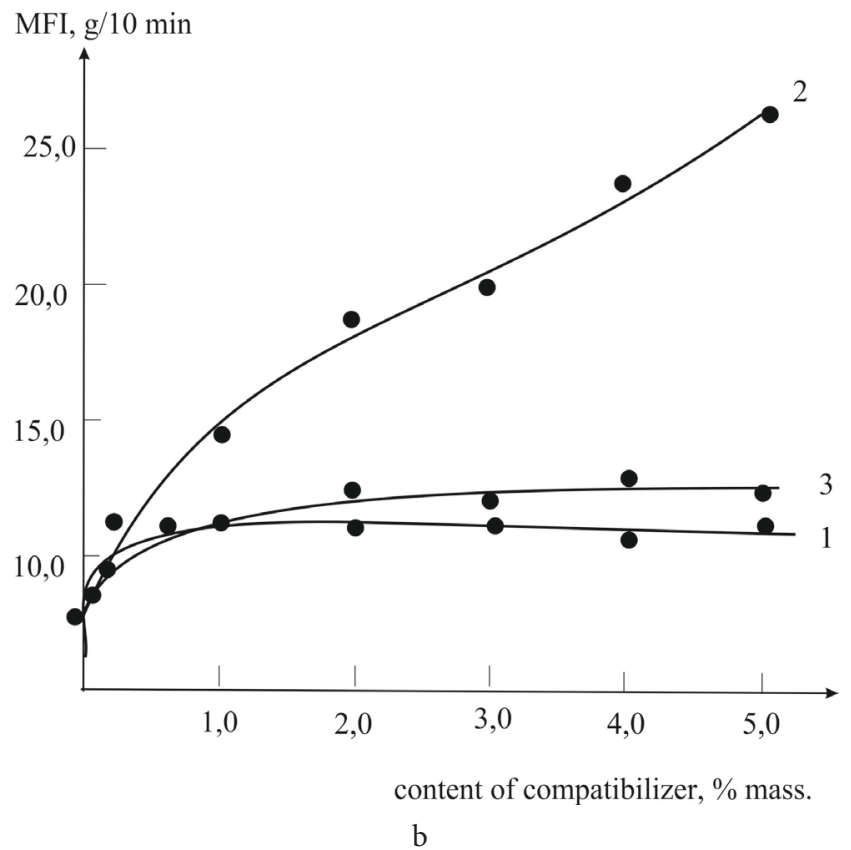

Fig. 1. The values of MFI for compositions based of the SPP containing $10 \mathrm{phr}$ of groundwood (a) and rice hulls (b) from the content of Kompplen EN13F1F3HH (1), Kompplen MPP5X (2) and San UMB UGOF1 (3) in the composition. 
into articles, it is safe to suggest that extending with compatibilizers increasing MFI brings an improvement in processing the composites by injection molding.

The data on MFI estimation are verified by the absolute rheometry data. Viscosity fluctuation behavior is similar to the MFI one meaning that when using Kompplen MPP5X as a compatibilizer, viscosity drops throughout the region of variation of compatibilizer content in the composition. In case of using Kompplen EN13F1F3HH and San UMB UGOF1 as compatibilizers viscosity only decreases in the region of low compatibilizer content, which is followed by a slight increase of viscosity (not over $30 \%$ ).

Of a great importance are not only viscosity values but also the polymer viscoelastic behaviour. It is well known [15] that polymer melts behave like viscoelastic fluids, for which not only viscous properties (flowability) but also elastic properties (recoverable deflection ability and energy storage) are characteristic. There are a lot of models to describe viscoelastic fluids properties, the Maxwell model predicting intensification of elastic properties of the polymer melt with the increase in shear rate being the simplest. As evidenced by the analysis of the data obtained, the loss modulus at low rates is greater that the storage modulus, while at high rates it is, on the contrary, smaller as follows from the Maxwell model. Threshold speed $\omega^{*}\left(120\right.$ and $260 \mathrm{~s}^{-1}$ for a composition based on SPP and 10 phr. of groundwood with and without Kompplen EN13F1F3HH, respectively) can be determined for the storage modulus to be greater than the loss modulus, which means that the polymer melt behaves like a substance with elastic properties prevailing over viscous ones. Since exhibiting elastic properties is what often results in defects, the higher the shear rate values in the process, the better. It was found that adding even a small amount of a compatibilizer results in the increase of $\omega^{*}$, which means that elastic properties of the composition containing a modifier are less pronounced, which is significantly positive.

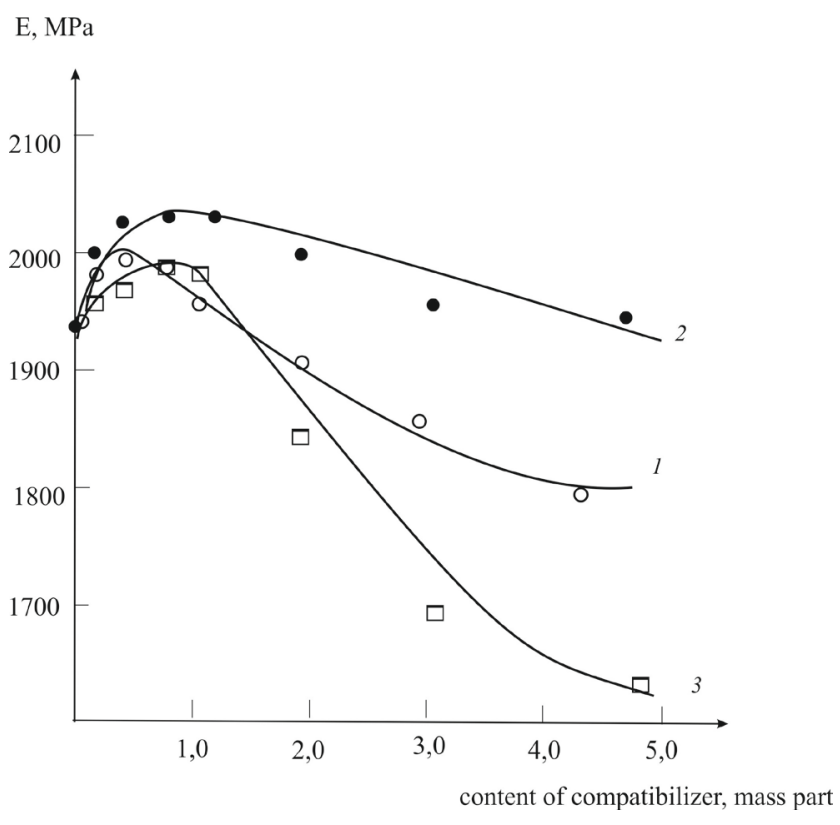

a
Changing the compatibilizer or the extender used does not result in the change of the general tendency for elastic properties exhibited by the polymer melt in the presence of the compatibilizer to weaken. The fact states that a composition extended with a modifier has a better workability.

It is important that the established regularities in the rheological behavior of the polymer-extender-modifier system have an effect on physico-mechanical properties of the materials (Table 1).

The aggregate data on elastic modulus changes are shown in Fig. 2.

As is seen from the data presented, the type of the extender has some effect on the ability of the polymer to resist ultimate tensile stress. The greatest differences in the effect of the modifiers for the compounds extended with RH and GW display themselves at the high content of the modifier. At its low content (about 1\%) the differences between the effects of the modifiers on the physico-mechanical properties of the materials are minimal. The effects of San UMB UGOF1 and Kompplen EN13F1F3HM modifiers are similar, while for

Table 1. Strain-strength properties of the composition based on the SPP containing $30 \mathrm{phr}$ of rice hulls and $0.5 \%$ of San UMB UGOF1 modifier obtained under various pressing conditions

\begin{tabular}{|c|c|c|c|}
\hline $\begin{array}{c}\text { Pressing } \\
\text { mode }\end{array}$ & $\begin{array}{c}\text { Elastic } \\
\text { modulus, MPa }\end{array}$ & $\begin{array}{c}\text { Ultimate tensile } \\
\text { stress, MPa }\end{array}$ & $\begin{array}{c}\text { Strain to } \\
\text { failure, \% }\end{array}$ \\
\hline 1 & 2020.6 & 11.7 & 4.9 \\
\hline 2 & 2162.6 & 16.3 & 5.3 \\
\hline 3 & 1432.1 & 11.5 & 6.0 \\
\hline 4 & 1881.5 & 13.4 & 4.6 \\
\hline 5 & 2100.5 & 15.0 & 5.2 \\
\hline 6 & 2175.0 & 15.4 & 5.2 \\
\hline 7 & 1547.5 & 9.7 & 5.7 \\
\hline 8 & 1876.8 & 14.7 & 5.2 \\
\hline
\end{tabular}

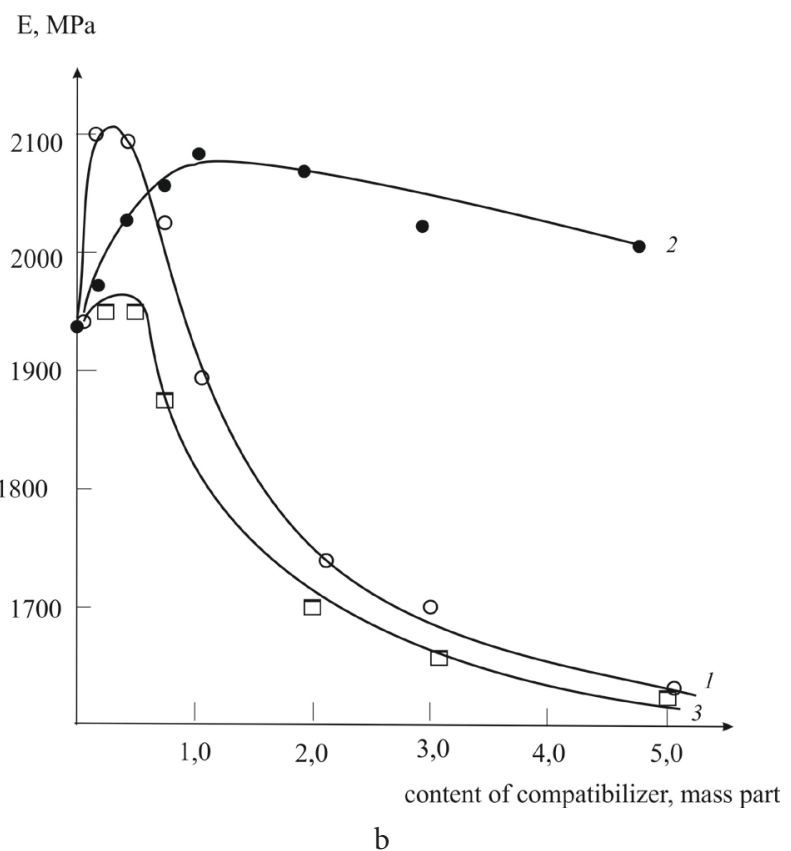

Fig. 2. Dependence of the elastic modulus of the composition based on SPP extended with $10 \mathrm{phr}$ of ground wood (a) and rice hulls (b) on the content of compatibilizers Kompplen EN13F1F3HH (1), Kompplen MPP5X (2) and San UMB UGOF1 (3) in the composition. 
Kompplen MPP5X it differs. It appears to be the result of the difference in chemical nature of the modifiers used.

The least values of the elastic modulus are registered when using San UMB UGOF1 as a modifier. In such a case the increased content of the extender in the compound decreases the already low values of the elastic modulus. For example, a compound consisting of SPP, $10 \mathrm{phr}$ of RH and $0.5 \%$ wt. of San UMB UGOF1, has the elastic modulus value of $1955 \mathrm{MPa}$. The values of ultimate tensile stress and strain to failure amount to $19.0 \mathrm{MPa}$ and $6.3 \%$, respectively. But the compounds obtained under the same conditions and containing $30 \mathrm{phr}$. of $\mathrm{RH}$ have the elastic modulus value of $1432 \mathrm{MPa}$, the ultimate tensile stress value of $11.5 \mathrm{MPa}$ and the strain to failure of $6.0 \%$, that is possess worse physicomechanical properties.

It is possible to significantly improve the strain-strength properties of a highly loaded polymer compound (Table 1) by changing the conditions of its formation.

The analysis of the data presented in the table supports the conclusion that cooling in the pressing process and reducing the operating temperature (i.e. accelerating the crystallization process of polypropylene) are accompanied by the increase in the values of the elastic modulus and ultimate tensile stress in the cases studied. This implies that varying pressing conditions makes it possible to obtain high-performance compositions even at high content of the extender.

As can be seen from the study, there is a possibility to vary rheological and physico-mechanical properties of polymer composites not only by using extenders acting as surfaceactive substances but also by adjusting the production conditions.

Aknowledgements. This paper was prepared as a part of the research work at Bashkir State University with financial support from the Ministry of Education and Science of the Russian Federation (Contract \# 03.G25.31.0275).

\section{References}

1. O. Faruk, A.K. Bledzki, H. Fink. Prog. Polym. Sci. 11, 1552 (2012). DOI: 10.1016/j.progpolymsci.2012.04.003

2. A. Boudenne, L. Ibos, Y. Candau, S. Thomas. Handbook of multiphase polymer systems. John Wiley and Sons Ltd, Chichester (2011).

3. I. A. Avilova, V.M. Buznik, V.I. Volkov, G. F. Zhelezina, E. V. Morozov, A.E. Raskutin, O. V. Falaleev. Aviation materials and technologies. S1, 30 (2014). (in Russian)

4. A. K. Mohanty, M. Misra, L. T. Drzal. Natural Fibers, Biopolymers and Biocomposites. Taylor\&Francis Group, USA (2005).

5. O. Faruk, M. Sain. Biofiber Reinforcements in Composite Materials. Cambridge, Woodhead Publishing Ltd. (2015) $772 \mathrm{p}$.

6. J. Jose, A. Nag, G. B. Nando. Iran Polym J. 23(10), 801 (2014).

7. R. Chandra, R. Rustgi. Prog. Polym. Sci. 23, 1273 (1998).

8. C. David, C. De Kesei, F. Lefebvre, W. Weiland. Angew Makromol Chem. 216, 21 (1994).

9. A. Arkatkar, J. Arutchelvi, M. Sudhakar. The Open Envir. Eng. J. 2, 68 (2009).

10. D. Garlotta. J Polym Environ. 9(2), 63 (2001).

11. Z. Lu John, Wu Qinglin, S. McNabb Harold. Wood and Fiber Science. 1, 88 (2000). DOI: 2-s2.0-0002538571

12. Yu. V. Kornev, A.M. Bukanov, O.B. Yumashev, V.A. Zhogin, Yu. A. Gamlitsky. The MITHT bulletin of M. V. Lomonosov. 4(2), 19 (2009). (in Russian)

13. E. L. Kalinchev, M.B. Sakovtseva. International news of the world of plastic. 7, 48 (2005) (in Russian)

14. V.I. Shaybakova, L.A. Abdrakhmanova, R. K. Nizamov. Polymers in construction: scientific Online magazine. 1(5), 46 (2017). (in Russian)

15. G. Schramm. A practical approach to rheology and rheometry. Koloss, Moskva (2003) 312 p. (in Russian) 